\title{
Neighbouring-group Influence on the Ring Opening of 2-Aryloxymethyl-1,1,2-tribromocyclopropanes under Phase-transfer Conditions
}

\author{
Einar Bakstad, ${ }^{a, b}$ Are S. Olsen, ${ }^{a}$ Marcel Sandberg ${ }^{a}$ and Leiv K. Sydnes ${ }^{a, b, *}$ \\ ${ }^{a}$ Department of Chemistry, University of Bergen, Allégt. 41, N-5007 Bergen, Norway and bepartment of Chemistry, \\ University of Troms $\varnothing$, N-9037 Troms $\varnothing$, Norway
}

\begin{abstract}
Bakstad, E., Olsen, A. S., Sandberg, M. and Sydnes, L. K., 1999. Neighbouringgroup Influence on the Ring Opening of 2-Aryloxymethyl-1,1,2-tribromocyclopropanes under Phase-transfer Conditions - Acta Chem. Scand. 53: 465-472. (c) Acta Chemica Scandinavica 1999.

When a number of 2-aryloxymethyl-1,1,2-tribromocyclopropanes were treated with sodium hydroxide and ethanol under phase-transfer conditions, ring opening occurred to give mixtures of acetylenic diethyl acetals and ketals in better than $80 \%$ total isolated yield. The acetylenic diethyl ketals predominated significantly and were, in some cases, almost the exclusive product. It is argued that this ketal selectivity is in part caused by hydrogen bonding between ethanol and the aryloxy group.
\end{abstract}

We have previously reported that 1,1,2-trihalocyclopropanes undergo ring opening when exposed to basic conditions and afford product mixtures, the composition of which is sensitive to the base employed. Thus, whereas sodium ethoxide in dry THF gives acetylenic diethyl ketals exclusively, 1,8-diazabicyclo[5.4.0]undec-7-ene (DBU) in ethanol converts the same substrates into acetylenic diethyl acetals only. On the other hand, when reacted with $50 \%$ aqueous sodium hydroxide in the presence of ethanol, triethylbenzylammonium chloride (TEBA) and dichloromethane (phase-transfer conditions, PTC), the trihalocyclopropanes give mixtures of ketal and acetal. ${ }^{1}$ When reacted under PTC, but employing isopropyl alcohol instead of ethanol, 1,1,2tribromocyclopropanes afford the corresponding acetylenic diisopropyl ketals only. ${ }^{2}$

The trends summarised above emerged from experiments with a selection of methyl- and phenyl-substituted 1,1,2-trihalocyclopropanes, i.e. substrates containing substituents with little variation in electronic and steric influence on the intermediates identified and confirmed to be involved in the reaction. It was therefore of interest to investigate the outcome of reactions of 1,1,2-trihalocyclopropanes containing substituents of higher polarity. The first prepared compound for this purpose was 1,1,2tribromo-2-(phenoxymethyl)cyclopropane, and when this tribromide was reacted under PTC in the presence of ethanol an almost regiospecific ring opening occurred. This observation prompted us to study base-induced ring

* Author to whom correspondence should be addressed at the University of Bergen. E-mail: leiv.sydnes@kj.uib.no opening of some similar compounds, and the results of this investigation are reported here.

\section{Results and discussion}

Preparation of 1,1,2-tribromocyclopropanes. The cyclopropanes used were prepared from the corresponding 3substituted 2-bromopropenes (1) which were synthesised from 2,3-dibromopropene by nucleophilic substitution reactions. The 3-aryloxy-2-bromopropenes (1a-1e) were made by generating the nucleophiles from the corresponding phenols with potassium carbonate in refluxing acetone, ${ }^{3-6}$ whereas 3-arylamino-2-bromopropenes if and $1 \mathrm{~g}$ and 3-phenylthio-2-bromopropene (1h) were obtained by treating 2,3-dibromopropene with mixtures of the corresponding amine/thiophenol and potassium carbonate in dimethyl sulfoxide at room temperature. The yield was excellent $(87-95 \%)$ in all cases.

Treatment of alkenes 1 with dibromocarbene gave the corresponding 1,1,2-tribromocyclopropanes in variable yields. Under phase-transfer conditions, in the absence of ethanol, ${ }^{7,8}$ the desired conversion took place with the 3-aryloxy-substituted alkenes (1a-1e) only, giving cyclopropanes 2a-2e (Scheme 1) in low to moderate yields (Table 1) under the best conditions ( 8 equivalents of bromoform and 6 equivalents of sodium hydroxide). The low yields were not caused by by-product formation since considerable amounts of unreacted alkene were recovered, but by a combination of the intrinsic alkene reactivity and the conditions prevailing during the cyclopropanation. This was borne out by the results obtained 


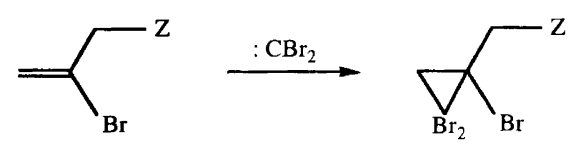

Scheme 1

$\begin{array}{lll}\text { 1 } & \mathrm{Z} & \mathbf{2} \\ \text { a } & \mathrm{PhO} & \text { a } \\ \text { b } & 4-\mathrm{MC}_{6} \mathrm{H}_{4} \mathrm{O} & \text { b } \\ \text { c } & 4-\mathrm{MeOC}_{6} \mathrm{H}_{4} \mathrm{O} & \text { c } \\ \text { d } & 4-\mathrm{ClC}_{6} \mathrm{H}_{4} \mathrm{O} & \text { d } \\ \text { e } & 4-\mathrm{O}_{2} \mathrm{NC}_{6} \mathrm{H}_{4} \mathrm{O} & \text { e } \\ \text { f } & 4-\mathrm{MeOC}_{6} \mathrm{H}_{4} \mathrm{NH} & \\ \text { g } & 4-\mathrm{MeOC}_{6} \mathrm{H}_{4} \mathrm{NBz} & \\ \text { h } & \mathrm{PhS} & \end{array}$

Table 1. 2-Aryloxymethyl-1,1,2-tribromocyclopropanes obtained by reacting dibromocarbene with alkenes 1 .

\begin{tabular}{llll}
\hline Alkene & Cyclopropane & Method & Isolated yield (\%) \\
\hline 1a & 2a & Makosza $^{a}$ & 42 \\
1b & 2b & Makosza $^{b}$ & 67 \\
1c & 2c & Makosza $^{b}$ & 49 \\
1d & 2d & Makosza $^{b}$ & 28 \\
& 2d & Seyferth $^{c}$ & 84 \\
1e & 2e & Makosza $^{a}$ & 10 \\
& 2e & Seyferth $^{c}$ & 79 \\
\hline
\end{tabular}

${ }^{a}$ At $25^{\circ} \mathrm{C} .{ }^{b}$ At $50-55^{\circ} \mathrm{C}$. ${ }^{c}$ At $80^{\circ} \mathrm{C}$.

by reacting 1d and 1e with dibromocarbene generated thermally from phenyl(tribromomethyl)mercury; ${ }^{9,10}$ the yields of $\mathbf{2 d}$ and $2 \mathrm{e}$ increased from low to very good (Table 1). Similar improvements have been reported by Müller and Nguyen-Thi. ${ }^{11}$

When alkenes $\mathbf{1 f}-\mathbf{1 h}$ were reacted with dibromocarbene under phase-transfer conditions the corresponding cyclopropanes were not obtained. In the case of 2-bromo-3phenylthiopropene (1h) the corresponding cyclopropane was most likely formed, but the compound was unstable and decomposed, giving an intractable, tarry, black product mixture. The alkene moiety of amines $1 \mathbf{f}$ and $\mathbf{1 g}$, on the other hand, did not react under these conditions; the former amine suffered $\mathrm{N}-\mathrm{H}$ insertion and gave 2-bromo-3-[ $N$-formyl- $N$-(4-methoxyphenyl) amino]propene as the final product in good yield $(81 \%)$ whereas $1 \mathrm{~g}$ was unreactive and was recovered almost quantitatively even after being stirred at elevated temperatures for an extended period of time. The observed insertion reaction has abundant literature precedence. ${ }^{12-14}$

The spectroscopic properties of the new compounds isolated were as expected. The ${ }^{1} \mathrm{H}$ NMR spectra of $\mathbf{2 a - 2 e}$ even exhibited coupling $(J 0.4 \mathrm{~Hz})$ between one of the cyclopropyl protons and one of the methylene protons next to the ring, a long-range coupling displayed by a number of gem-dihalocyclopropanes. ${ }^{15,16}$

Ring opening. Treatment of the cyclopropanes with sodium hydroxide under phase-transfer conditions in the presence of ethanol gave product mixtures, whose composition was sensitive to the substituent attached to the phenyl group. When $\mathbf{2 a - 2 d}$ were reacted, the acetylenic diethyl ketal 3 and the corresponding acetylenic diethyl acetal 4 were formed almost exclusively (Scheme 2). In all cases the total isolated yield was better than $80 \%$, with the ketal always predominating, but the amount of 3 relative to 4 was strongly influenced by the substituent attached to the phenyl group (Table 2). When the phenyl ring was methyl-substituted in the para position $(\mathbf{2 b})$ the $3 / 4$ ratio was 7 , which is significantly lower than the ratio of 40 observed when the chloro-substituted analogue (2d) was reacted under identical conditions.

The substituent influence was most profound in 1,1,2tribromo-2-(4-nitrophenoxymethyl)cyclopropane (2e) which afforded a black, intractable product mixture consisting of a large number of compounds when exposed to sodium hydroxide under the standard conditions. Not a single compound could be isolated pure although a number of techniques were tried, but when the IR and ${ }^{1} \mathrm{H}$ NMR spectra of the crude reaction mixture were compared with the spectra of pure samples of $\mathbf{3 a}-\mathbf{3} \mathbf{d}$ and $\mathbf{4 a}-\mathbf{4 d}$, the presence of acetylenic diethyl ketal $3 \mathbf{e}$ and acetylenic diethyl acetal $\mathbf{4 e}$ was confirmed. Thus, in addition to characteristic signals due to the gem-diethoxy moiety, IR absorptions at 3275 and $2120 \mathrm{~cm}^{-1}$ and a singlet at $2.52 \mathrm{ppm}$ in the proton NMR spectrum indicated the presence of $3 \mathbf{e}$, whereas a triplet at $5.14 \mathrm{ppm}$ and a doublet at $4.81 \mathrm{ppm}$, with a coupling constant of $1.4 \mathrm{~Hz}$ and a relative area of $1: 2$, are the expected signals for the methine and methylene protons, respectively, of 4e. By integration of appropriate NMR signals the $3 / 4$ ratio was determined to be 2.5 .

Table 2. Acetylenic diethyl ketals (3) and acetals (4) obtained by reacting 2 with sodium hydroxide under phase-transfer conditions.

\begin{tabular}{llcl}
\hline Cyclopropane & Products & $\mathbf{3 : 4}$ & Total yield of $\mathbf{3}$ and $\mathbf{4}(\%)$ \\
\hline $\mathbf{2 a}$ & $\mathbf{3 a}+\mathbf{4 a}$ & 18 & 95 \\
$\mathbf{2 b}$ & $\mathbf{3 b}+\mathbf{4 b}$ & 7 & 83 \\
$\mathbf{2 c}$ & $\mathbf{3 c}+\mathbf{4 c}$ & 8 & 89 \\
$\mathbf{2 d}$ & $\mathbf{3 d}+\mathbf{4 d}$ & 40 & 82 \\
$\mathbf{2 e}$ & $\mathbf{3 e}+\mathbf{4} \mathbf{e}^{\mathbf{a}}$ & 2.5 & - \\
\hline
\end{tabular}

${ }^{a} A$ complex reaction mixture which also contained $3 \mathbf{e}$ and $4 \mathrm{e}$ (see the text), was obtained.

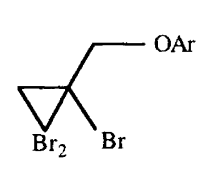

$\mathbf{2 a}-\mathbf{2 e}$

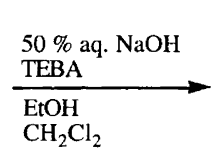

$\mathrm{CH}_{2} \mathrm{Cl}_{2}$

Scheme 2.

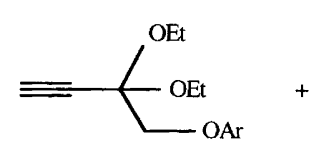

3

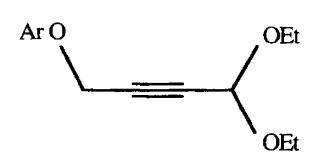

4 


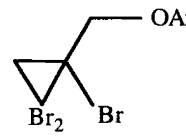

$\mathbf{2 a}-\mathbf{2 e}$
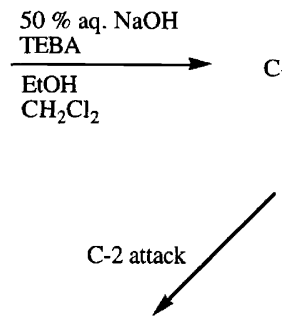

3

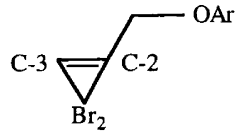

5<smiles>[GeH3]</smiles>

4
Scheme 3.

Considering the fact the ketal/acetal ratio is $1.0-1.5$ when methyl- and phenyl-substituted 1,1,2-tribromocyclopropanes react with sodium hydroxide as described for tribromides $\mathbf{2}$, the most characteristic feature of the ring-opening reactions presented above is the overwhelming acetylenic-ketal predominance. Introduction of an aryloxymethyl moiety at the expense of a methyl or a phenyl group obviously makes ketal formation much more favourable than acetal formation. Consequently, the double bond in cyclopropene $\mathbf{5}$, assumed to be formed from 2 as an intermediate during the reaction, ${ }^{1}$ is attacked almost exclusively at the more substituted carbon atom (C-2) (Scheme 3 ) by both ethoxide and ethanol. From previous results such a selective attack by ethoxide might be anticipated, ${ }^{2}$ and indeed, when $2 \mathrm{c}$ was reacted with sodium ethoxide, prepared from sodium and absolutely dry ethanol, in dry $\mathrm{THF},{ }^{2}$ regiospecific attack of $\mathrm{C}-2$, giving the corresponding diethyl ketal (3c), was achieved. However, selective attack of C-2 by ethanol is surprising considering the C-3 selectivity previously observed for this alcohol ${ }^{2}$ as well as the larger steric bulk of the aryloxymethyl groups compared to the methyl group. These facts, therefore, indicate that one or several other effects, which offset the steric interactions and redirect the predominant attack of ethanol from C-3 to C-2, are involved and lead to the observed significant ketal preference.

Owing to the distance between the aryl group and the cyclopropene carbon-carbon double bond, electronic through-bond interactions are unlikely to play a significant role in the redirection of the ethanol attack. If such interactions were important, a systematic variation in the 3:4 ratio would have been expected when the substituent attached to the phenyl group was varied, but this was not observed. Another possible mechanism involves a cyclopropenyl-cation intermediate, ${ }^{17,18}$ which may be generated from cyclopropenes like 5 by expulsion of a bromide ion. If such an intermediate were involved, however, one would, by analogy, expect products to be formed from nucleophilic attack at all the three ringcarbon atoms. ${ }^{17,18}$ Such extensive product formation was not observed in any of our reactions, and this renders cyclopropenyl cations unlikely as intermediates.

A final option, which explains the ketal predominance in the ring opening of 2 , is that redirection of the ethanol attack of the intermediate cyclopropene from C-3 to C2 is caused by hydrogen bonding between the attacking alcohol molecule and the oxygen atom of the aryloxy group. The existence of such a directing interaction is supported by several observations. Firstly, 2c, when refluxed in a mixture of ethanol and $\mathrm{DBU}$, reaction conditions which lead to regiospecific attack at C-3 and exclusive formation of acetylenic acetals when methyl- and phenyl-substituted trihalocyclopropanes are reacted, ${ }^{2}$ also gave a minor amount of ketal $3 \mathbf{c}$ as revealed by the presence of appropriate peaks in the IR and ${ }^{1} \mathrm{H}$ NMR spectra of the reaction mixture (see the Experimental). Furthermore, when $2 \mathrm{c}$ was reacted with sodium hydroxide in the presence of isopropyl alcohol under PTC, reaction conditions which also lead to regiospecific attack at C-3 and exclusive formation of acetylenic acetals when methyl- and phenyl-substituted trihalocyclopropanes are reacted, ${ }^{2}$ the acetal [1,1-diisopropoxy-4-(4methoxyphenoxy)-2-butyne, 6] predominated, but the corresponding ketal [3,3-diisopropoxy-4-(4-methoxyphenoxy)-1-butyne, 7] was also formed $(6: 7=19: 1)$ (Scheme 4). The most convincing evidence, however, was obtained when 1,1,2-trichloro-2-diethoxymethyl-3-

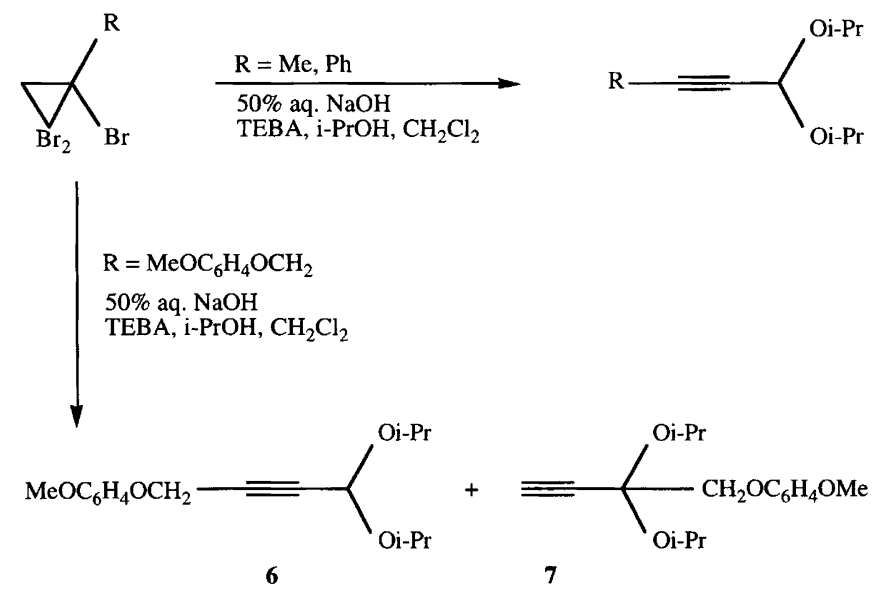

Scheme 4. 
methylcyclopropane (8) was allowed to react under PTC in the presence of ethanol. The reaction proceeded smoothly and gave a reaction mixture which consisted mainly of 4,4,5,5-tetraethoxy-2-pentyne (9) and contained none of the isomeric acetylene 1,1,4,4-tetraethoxy-2-pentyne (10) (Scheme 5). The intermediate cyclopropene has therefore been attacked exclusively at C-2 in spite of the fact that this carbon atom is sterically less accessible than C-3. It is therefore proposed that the specificity is caused by hydrogen-bonding-directed attack of ethanol at C-2.

\section{Experimental}

General. IR spectra were recorded on Perkin Elmer 1310 and Nicolet 400 infrared spectrophotometers. NMR spectra were run on Jeol FX 90Q, Bruker Spectrospin AC $200 \mathrm{~F}$ spectrometer and Bruker Spectrospin DMX 400 instruments with $\mathrm{CDCl}_{3}$ as the solvent unless otherwise stated, and tetramethylsilane (TMS) as an internal reference. Chemical shifts are reported in ppm downfield from TMS. Mass spectra were obtained on a VG Fisons $7070 \mathrm{E}$ Micromass spectrometer operated in the EI mode at $70 \mathrm{eV}$. GC analyses were carried out on a Hewlett Packard 5720A gas chromatograph equipped with FID and a $15 \%$ SP 2100 packed silica column. TLC analyses of the reaction mixtures were carried out with silica gel $60 \mathrm{~F}_{254}$ on aluminium sheets (Merck No. 5554) with hexane-ethyl acetate as the solvent. Flash chromatography was carried out on a $2 \mathrm{~cm} \times 70 \mathrm{~cm}$ column with silica gel (Merck No. 9385) as the stationary phase and the mobile phase as indicated in each case. Silica gel (Merck No. 9385) was also used as the stationary phase in dry-flash chromatography.

Chemicals. THF was distilled from sodium-benzophenone ketyl under nitrogen immediately prior to use. Benzene was dried over sodium wire. DMSO was distilled from calcium hydride and stored over molecular sieves $4 \AA$. Acetone was dried with anhydrous potassium carbonate, distilled and stored over molecular sieves $4 \AA$.

Preparation of 3-aryloxy-2-bromopropenes 1a-1e; general procedure. To a mixture of a phenol $(0.20 \mathrm{~mol})$ and anhydrous potassium carbonate $(27.64 \mathrm{~g}, 0.20 \mathrm{~mol})$ in anhydrous acetone $(200 \mathrm{ml})$ kept under nitrogen, was added 2,3-dibromopropene (39.97 g, $0.20 \mathrm{~mol}$ ) prepared from allyl bromide. The resulting mixture was stirred vigorously, refluxed and monitored by GC and TLC (approximately $15 \mathrm{~h}$ ). Water was added, and the products were extracted with diethyl ether, worked up in the usual way and isolated by distillation or recrystallisation.

2-Bromo-3-phenoxypropene (1a), from phenol, b.p. $59-60^{\circ} \mathrm{C} / 0.4 \mathrm{mmHg}$ (lit. ${ }^{19}$ b.p. $98-99^{\circ} \mathrm{C} / 2.5 \mathrm{mmHg}$ ); yield: $39.0 \mathrm{~g}(92 \%)$.

2-Bromo-3-(4-methylphenoxy) propene (1b), from 4methylphenol, b.p. $70-71^{\circ} \mathrm{C} / 1 \mathrm{mmHg}$; yield: $42.7 \mathrm{~g}$ (94\%). IR (film): 3020 (m), $2920(\mathrm{~m}), 2860$ (m), 1695 $(\mathrm{w}), 1630(\mathrm{~m}), 1605(\mathrm{~m}), 1580(\mathrm{~m}), 1505(\mathrm{~s}), 1445(\mathrm{~m})$, $1375(\mathrm{~m}), 1290(\mathrm{~m}), 1230(\mathrm{~s}), 1210(\mathrm{~s}), 1170(\mathrm{~m}), 1115$ (m), $1040(\mathrm{~s}), 895(\mathrm{~m}), 845(\mathrm{w}), 820(\mathrm{~s}), 760(\mathrm{w}), 720$ (w) $\mathrm{cm}^{-1} \cdot{ }^{1} \mathrm{H}$ NMR (200 MHz): $\delta \quad 2.27 \quad(3 \mathrm{H}, \mathrm{s})$, 4.56-4.58 (2 H, m), 5.61-5.64 (1 H, m), 5.94-5.97 $(1 \mathrm{H}, \mathrm{m}), 6.75-6.83(2 \mathrm{H}, \mathrm{m}), 7.02-7.10(2 \mathrm{H}, \mathrm{m}) .{ }^{13} \mathrm{C}$ NMR $(50 \mathrm{MHz}): \delta 20.3\left(\mathrm{CH}_{3}\right), 71.6\left(\mathrm{OCH}_{2}\right), 114.6$ $(2 \times \mathrm{CH}), 117.4\left(=\mathrm{CH}_{2}\right), 127.2(\mathrm{CBr}), 129.8(2 \times \mathrm{CH})$, 130.6 (C), 155.5 (C).

2-Bromo-3-(4-methoxyphenoxy) propene (1c), from 4methoxyphenol, b.p. $81 \cdot 83^{\circ} \mathrm{C}^{\prime} 0.05 \mathrm{mmHg}$; yield: $42.8 \mathrm{~g}$ $(88 \%)$. IR (film): 3045 (w), $3010(w), 2960$ (m), 2930 (m), $2910(\mathrm{~m}), 2830(\mathrm{~m}), 2060 \mathrm{w}), 1650(\mathrm{~m}), 1600(\mathrm{w})$, $1510(\mathrm{~s}), 1450(\mathrm{~m}), 1305(\mathrm{~m}), 1240(\mathrm{~s}), 1180(\mathrm{~m}), 1110$ (m), $1050(\mathrm{~s}), 905(\mathrm{~m}), 830(\mathrm{~s}), 760(\mathrm{~m}), 730(\mathrm{~m}) \mathrm{cm}^{-1}$. ${ }^{1} \mathrm{H}$ NMR (200 MHz): $\delta 3.76(3 \mathrm{H}, \mathrm{s}), 4.57-4.59(2 \mathrm{H}, \mathrm{m})$, 5.64-5.66 (1 H, m), 5.96-5.99 (1 H, m), 6.79-6.89 $(4 \mathrm{H}, \mathrm{m}),{ }^{13} \mathrm{C}$ NMR $(50 \mathrm{MHz}): \delta 55.5\left(\mathrm{OCH}_{3}\right), 72.5$ $\left(\mathrm{OCH}_{2}\right), 114.2(2 \times \mathrm{CH}), 115.9(2 \times \mathrm{CH}), 117.6\left(=\mathrm{CH}_{2}\right)$, $127.4(\mathrm{CBr}), 151.8(\mathrm{C}), 154.3(\mathrm{C})$.

2-Bromo-3-(4-chlorophenoxy) propene (1d), from 4-chlorophenol, b.p. $130-132{ }^{\circ} \mathrm{C} / 10 \mathrm{mmHg}$; yield: $47.0 \mathrm{~g} \mathrm{(95 \% )}$. IR (film): 3100 (w), 3070 (w), 2920 (w), 2860 (w), 1890 (w), 1650 (m), 1590 (m), 1490 (s), 1305 (s), 1235 (s), 1110 (s), 1050 (s), 910 (s), 830 (s), 700 (m) cm ${ }^{-1} \cdot{ }^{1} \mathrm{H}$ NMR (200 MHz): $\delta$ 4.59-4.67 (2 H, m), 5.66-5.68 $(1 \mathrm{H}, \mathrm{m}), \quad 5.95-5.98 \quad(1 \mathrm{H}, \mathrm{m}), \quad 6.80-6.88 \quad(2 \mathrm{H}, \mathrm{m})$, $7.20-7.28(2 \mathrm{H}, \mathrm{m}) .{ }^{13} \mathrm{C} \mathrm{NMR}(50 \mathrm{MHz}): \delta 71.8\left(\mathrm{OCH}_{2}\right)$, $116.1(2 \times \mathrm{CH}), 117.9\left(=\mathrm{CH}_{2}\right), 126.4(\mathrm{C}), 126.5(\mathrm{CBr})$, $129.3(2 \times \mathrm{CH}), 152.6(\mathrm{C})$.

2-Bromo-3-(4-nitrophenoxy) propene (1e), from 4-nitrophenol, m.p. $37-38{ }^{\circ} \mathrm{C}$; yield: $44.9 \mathrm{~g}(87 \%)$. IR $\left(\mathrm{CHCl}_{3}\right)$ : $3070(\mathrm{w}), 3010(\mathrm{~m}), 2915(\mathrm{w}), 1940(\mathrm{w}), 1605(\mathrm{~s}), 1585$ (s), $1505(\mathrm{~s}), 1490(\mathrm{~s}), 1445(\mathrm{~m}), 1340(\mathrm{~s}), 1290(\mathrm{~m}), 1255$ (s), $1215(\mathrm{~s}$ broad $), 1170(\mathrm{~m}), 1110(\mathrm{~s}), 1030(\mathrm{~s}), 900(\mathrm{~m})$,

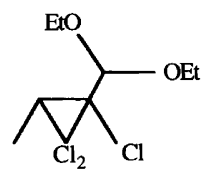

8

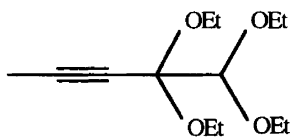

9

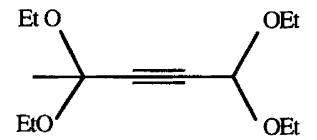

10

Scheme 5 . 
$860(\mathrm{~m}), 840(\mathrm{~s}) \mathrm{cm}^{-1} .{ }^{1} \mathrm{H}$ NMR $(200 \mathrm{MHz}): \delta 4.74-4.75$ $(2 \mathrm{H}, \mathrm{m}), \quad 5.73-5.75 \quad(1 \mathrm{H}, \mathrm{m}), \quad 5.99-6.02 \quad(1 \mathrm{H}, \mathrm{m})$, 6.96-7.04 (2 H, m), 8.17-8.26 (2 H, m). ${ }^{13} \mathrm{C}$ NMR $(50 \mathrm{MHz}): \quad \delta 71.7\left(\mathrm{OCH}_{2}\right), 114.7(2 \times \mathrm{CH}), 118.6$ $\left(=\mathrm{CH}_{2}\right), 125.3(\mathrm{CBr}), 125.8(2 \times \mathrm{CH}), 142.1(\mathrm{C}), 162.4$ (C). MS $\left[m / z\right.$ (rel. int.)]: $259\left(79, M^{+}\right), 257\left(81, M^{+}\right)$, 243 (12), 241 (12), 178 (43), 161 (7), 132 (100), 121 (37), 119 (38), $109(8), 93$ (6), 76 (5), 63 (16).

General procedure for the preparation of alkenes $\mathbf{1 f}-\mathbf{1 h}$. To a mixture of amine or thiophenol $(0.10 \mathrm{~mol})$, and anhydrous potassium carbonate $(13.82 \mathrm{~g}, 0.10 \mathrm{~mol})$ in anhydrous dimethyl sulfoxide $(150 \mathrm{ml})$ kept under nitrogen at $0{ }^{\circ} \mathrm{C}$ was added slowly a solution of 2,3-dibromopropene $(19.99 \mathrm{~g}, 0.10 \mathrm{~mol})$. When the addition was complete, the resulting mixture was stirred vigorously at room temperature and monitored by GC and TLC (approximately $15 \mathrm{~h})$. Water $(200 \mathrm{ml})$ was added, the product was extracted with hexane $(5 \times 75 \mathrm{ml})$, and the combined extracts were washed with water $(2 \times 100 \mathrm{ml})$ and dried $\left(\mathrm{MgSO}_{4}\right)$. Filtration and evaporation of the solvent left an oil which was essentially pure product.

2-Bromo-3-(4-methoxyphenylamino)propene (1f), from 4-methoxyaniline (12.32 g); yield: $21.3 \mathrm{~g}(88 \%)$. IR (film): 3390 (m), 2980 (m), 2940 (m), 2920 (m), 2890 (m), 2820 (m), $1715(\mathrm{w}), 1625(\mathrm{~m}), 1505(\mathrm{~s}), 1455(\mathrm{~m}), 1430(\mathrm{~m})$, $1230(\mathrm{~s}), 1170(\mathrm{~m}), 1105(\mathrm{~m}), 1065(\mathrm{~m}), 1030(\mathrm{~s}), 890$ (m), 815 (s) $\mathrm{cm}^{-1} .{ }^{1} \mathrm{H}$ NMR spectrum. ${ }^{20}{ }^{13} \mathrm{C}$ NMR $(50 \mathrm{MHz}): \delta 52.7\left(\mathrm{NCH}_{2}\right), 55.5\left(\mathrm{OCH}_{3}\right), 114.1(2 \times \mathrm{CH})$, $114.6(2 \times \mathrm{CH}), 116.5\left(=\mathrm{CH}_{2}\right), 131.4(\mathrm{CBr}), 140.6(\mathrm{C})$, $152.3(\mathrm{C})$.

\section{2-Bromo-3-[benzyl (4-methoxyphenyl)amino]propene (1g),} from $N$-benzyl-4-methoxyaniline; ${ }^{21}$ yield $30 \mathrm{~g}(90 \%)$. IR (film): 3055 (m), 3020 (m), 2920 (m), 2820 (m), $1720(\mathrm{w})$, $1625(\mathrm{~m}), 1510(\mathrm{~s}), 1450(\mathrm{~s}), 1355(\mathrm{~s}), 1235(\mathrm{~s}), 1105(\mathrm{~m})$, $1030(\mathrm{~s}), 955(\mathrm{~m}), 890(\mathrm{~m}), 810(\mathrm{~s}), 735(\mathrm{~s}), 695(\mathrm{~s}) \mathrm{cm}^{-1}$. ${ }^{1} \mathrm{H}$ NMR (200 MHz): $\delta 3.69(3 \mathrm{H}, \mathrm{s}), 4.12(2 \mathrm{H}, \mathrm{s}), 4.52$ $(2 \mathrm{H}, \mathrm{s}), \quad 5.54-5.57 \quad(1 \mathrm{H}, \mathrm{m}), \quad 5.72-5.75 \quad(1 \mathrm{H}, \mathrm{m})$, 6.55-6.78 (4 H, m), 7.15-7.32 (5 H, m). ${ }^{13} \mathrm{C} \quad \mathrm{NMR}$ $(50 \mathrm{MHz}): \delta 54.5\left(\mathrm{NCH}_{2}\right), 59.4\left(\mathrm{NCH}_{2}\right), 55.5\left(\mathrm{OCH}_{3}\right)$, $114.1(2 \times \mathrm{CH}), 114.6(2 \times \mathrm{CH}), 116.1\left(=\mathrm{CH}_{2}\right), 126.6$ $(2 \times \mathrm{CH}), 126.8(\mathrm{CH}), 128.4(2 \times \mathrm{CH}), 129.7(\mathrm{CBr})$, 138.3 (C), 142.1 (C), 151.9 (C).

2-Bromo-3-phenylthiopropene (1h), from thiophenol, b.p. $142-143{ }^{\circ} \mathrm{C} / 15 \mathrm{mmHg}$; yield: $20.1 \mathrm{~g}(88 \%)$. IR (film): 3060 (m), 3040 (m), 2900 (w), 1940 (w), 1785 (w), 1610 (m), $1570(\mathrm{~m}), 1465(\mathrm{~s}), 1425(\mathrm{~m}), 1395(\mathrm{~m}), 1295(\mathrm{w})$, $1235(\mathrm{~m}), 1190(\mathrm{~m}), 1080(\mathrm{~m}), 1060(\mathrm{~m}), 1015(\mathrm{~m}), 890$ (s), $740(\mathrm{~s}), 690(\mathrm{~s}), 610(\mathrm{~m}) \mathrm{cm}^{-1} .{ }^{1} \mathrm{H}$ NMR (200 MHz): $\delta$ 3.79-3.80 (2 H, m), 5.44-5.46 (1 H, m), 5.70-5.73 $(1 \mathrm{H}, \mathrm{m}), 7.20-7.39(5 \mathrm{H}, \mathrm{m}) .{ }^{13} \mathrm{C}$ NMR $(50 \mathrm{MHz}): \delta$ $44.6\left(\mathrm{SCH}_{2}\right), 118.9\left(=\mathrm{CH}_{2}\right), 127.3(\mathrm{CH}), 128.6(\mathrm{CBr})$, $128.9(2 \times \mathrm{CH}), 130.7(2 \times \mathrm{CH}), 134.6(\mathrm{C})$.

Preparation of trihalocyclopropanes; general procedure. To a vigorously stirred solution of bromoalkene 1
$(0.050 \mathrm{~mol})$, ethanol-free bromoform (101.1 g, $0.40 \mathrm{~mol}$ ), and triethylbenzylammonium chloride (TEBA, ca. $0.3 \mathrm{~g}$ ) was added dropwise a $50 \%$ aqueous solution of $\mathrm{NaOH}(24.0 \mathrm{~g}, 0.30 \mathrm{~mol})$. The resulting mixture was stirred overnight at room temperature (1a and 1e) or at $50-55^{\circ} \mathrm{C}(\mathbf{1 b}-\mathbf{1 d})$. Water $(150 \mathrm{ml})$ was added followed by hydrochloric acid $(6 \mathrm{M})$ until the solution became acidic. The product was extracted with dichloromethane $(4 \times 75 \mathrm{ml})$ and the combined extracts were dried $\left(\mathrm{MgSO}_{4}\right)$. Filtration and evaporation of the solvent gave a residue from which excess bromoform and unreacted starting material were removed by distillation under vacuum (at 20 and $0.1 \mathrm{mmHg}$, respectively). The product was isolated by flash chromatography and subsequently recrystallized. The results of the syntheses are summarized in Table 1.

1,1,2-Tribromo-2-phenoxymethylcyclopropane (2a) was obtained from 1a and isolated by flash chromatography (hexane) and subsequently recrystallized from hexane to give light yellow crystals; m.p. $44-46^{\circ} \mathrm{C}$; yield: $8.1 \mathrm{~g}$ $(42 \%)$. IR $\left(\mathrm{CCl}_{4}\right): 3050(\mathrm{w}), 3030(\mathrm{w}), 2915(\mathrm{w}), 1590$ (s), $1580(\mathrm{sh}), 1490(\mathrm{~s}), 1450(\mathrm{~m}), 1410(\mathrm{w}), 1380(\mathrm{~m})$, $1295(\mathrm{~m}), 1235$ (s), $1210(\mathrm{sh}), 1170(\mathrm{~m}), 1150(\mathrm{w}), 1075$ (m), $1050(\mathrm{~m}), 1010(\mathrm{~m}), 880(\mathrm{w}), 690(\mathrm{~m}) \mathrm{cm}^{-1} \cdot{ }^{1} \mathrm{H}$ NMR $(200 \mathrm{MHz}): \delta 2.03(1 \mathrm{H}$, dd, $J 9.5$ and $0.5 \mathrm{~Hz})$, $2.12(1 \mathrm{H}, \mathrm{d}, J 9.5 \mathrm{~Hz}), 4.41(1 \mathrm{H}, \mathrm{dd}, J 11.0$ and $0.5 \mathrm{~Hz})$, $4.50(1 \mathrm{H}, \mathrm{d}, J 11.0 \mathrm{~Hz}), 6.92-7.03(3 \mathrm{H}, \mathrm{m}), 7.24-7.34$ $(2 \mathrm{H}, \mathrm{m}) .{ }^{13} \mathrm{C} \mathrm{NMR}(22.5 \mathrm{MHz}): \delta 29.6\left(\mathrm{CBr}_{2}\right), 36.0$ $\left(\mathrm{CH}_{2}\right), 41.0(\mathrm{CBr}), 75.0\left(\mathrm{OCH}_{2}\right), 115.2(2 \times \mathrm{CH}), 121.8$ $(\mathrm{CH}), 129.5(2 \times \mathrm{CH}), 158.0(\mathrm{C})$. MS [ $\mathrm{m} / \mathrm{z}$ (rel. int.)]: $388\left(20, M^{+}\right), 386\left(61, M^{+}\right), 384\left(66, M^{+}\right), 382(21$, $M^{+}$), 305 (32), 291 (7), 212 (18), 131 (13), 94 (100), 77 (21), 65 (29), 51 (39).

1,1,2-Tribromo-2-(4-methylphenoxy) methylcyclopropane (2b) was obtained from $\mathbf{1 b}$ and was isolated by flash chromatography (hexane) and subsequently recrystallized from hexane to give light yellow crystals; m.p. 35-37 ${ }^{\circ} \mathrm{C}$; yield: $13.4 \mathrm{~g}(67 \%)$. IR $\left(\mathrm{CCl}_{4}\right): 3020(\mathrm{w}), 2915$ (m), $2860(\mathrm{w}), 1605(\mathrm{~m}), 1530(\mathrm{~m}), 1505(\mathrm{~s}), 1450(\mathrm{~m})$, 1380 (m), 1285 (m), 1235 (br, s), 1170 (m), 1055 (m), $1010(\mathrm{~m}), 975(\mathrm{w}), 930(\mathrm{w}), 695(\mathrm{~m}) \mathrm{cm}^{-1} .{ }^{1} \mathrm{H}$ NMR (200 MHz): $\delta 2.02(1 \mathrm{H}$, dd, $J 9.5$ and $0.4 \mathrm{~Hz}), 2.13$ $(1 \mathrm{H}, \mathrm{d}, J 9.5 \mathrm{~Hz}), 2.29(3 \mathrm{H}, \mathrm{s}), 4.36(1 \mathrm{H}, \mathrm{dd}, J 11.1$ and $0.4 \mathrm{~Hz}), 4.46(1 \mathrm{H}, \mathrm{d}, J 11.1 \mathrm{~Hz}), 6.83-6.87(2 \mathrm{H}, \mathrm{m})$, 7.07-7.11 (2 H, m). ${ }^{13} \mathrm{C} \mathrm{NMR} \mathrm{(50} \mathrm{MHz):} \delta 20.4\left(\mathrm{CH}_{3}\right)$, $29.5\left(\mathrm{CBr}_{2}\right), 35.9\left(\mathrm{CH}_{2}\right), 41.0(\mathrm{CBr}), 75.2\left(\mathrm{OCH}_{2}\right), 115.0$ $(2 \times \mathrm{CH}), 129.9(2 \times \mathrm{CH}), 131.0(\mathrm{C}), 155.9(\mathrm{C}) . \mathrm{MS}[\mathrm{m} / \mathrm{z}$ (rel. int.)]: $402\left(5, M^{+}\right), 400\left(18, M^{+}\right), 398\left(18, M^{+}\right)$, $396\left(5, M^{+}\right), 319(21), 291(9), 212(27), 131$ (11), 108 (100), 91 (7), 77 (11), 51 (11).

1,1,2 - Tribromo-2 - (4-methoxyphenoxy) methylcyclopro pane (2c) was obtained from $1 \mathbf{c}$ and was isolated by flash chromatography (hexane) and subsequently recrystallized from hexane to give light yellow crystals; m.p. 65-67 ${ }^{\circ} \mathrm{C}$; yield: $10.2 \mathrm{~g}(49 \%)$. IR $\left(\mathrm{CCl}_{4}\right): 2990(\mathrm{w}), 2920$ 
(br, w), 2820 (w), 1495 (m), 1500 (br, w), 1225 (br, s), $1175(w), 1100(w), 1040(\mathrm{~m}), 1010(w), 695(w) \mathrm{cm}^{-1}$. ${ }^{1} \mathrm{H}$ NMR $(400 \mathrm{MHz}): \delta 2.02(1 \mathrm{H}, \mathrm{dd}, J 9.5$ and $0.4 \mathrm{~Hz})$, $2.12(1 \mathrm{H}, \mathrm{d} J 9.5 \mathrm{~Hz}), 3.76(3 \mathrm{H}, \mathrm{s}), 4.36(1 \mathrm{H}, \mathrm{dd}, J$ 11.1 and $0.4 \mathrm{~Hz}), 4.44(1 \mathrm{H}, \mathrm{d}, J 11.1 \mathrm{~Hz}), 6.82-6.86$ (2 H, m), 6.89-6.93 (2 H, m). ${ }^{13} \mathrm{C}$ NMR $(50 \mathrm{MHz}): \delta$ $29.6\left(\mathrm{CBr}_{2}\right), 35.9\left(\mathrm{CH}_{2}\right), 41.1(\mathrm{CBr}), 55.6\left(\mathrm{OCH}_{3}\right), 76.0$ $\left(\mathrm{OCH}_{2}\right), 114.6(2 \times \mathrm{CH}), 116.5(2 \times \mathrm{CH}), 152.2(\mathrm{C})$, $154.6(\mathrm{C})$.

1,1,2-Tribromo-2-(4- chlorophenoxy) methylcyclopropane (2d) was obtained from 1d and was isolated by flash chromatography (hexane) and subsequently recrystallized from hexane to give yellow crystals; m.p. $69-71{ }^{\circ} \mathrm{C}$; yield: $5.9 \mathrm{~g}(28 \%)$. IR $\left(\mathrm{CCl}_{4}\right): 3070(\mathrm{w}), 2910(\mathrm{w}), 1590$ $(\mathrm{m}), 1575(\mathrm{~m}), 1485(\mathrm{~s}), 1450(\mathrm{~m}), 1380(\mathrm{~m}), 1275(\mathrm{~m})$, $1235(\mathrm{~s}), 1165(\mathrm{~m}), 1090(\mathrm{~m}), 1045(\mathrm{~m}), 1005(\mathrm{~m}), 820$ (m), $690(\mathrm{~m}), 670(\mathrm{~m}) \mathrm{cm}^{-1} .{ }^{1} \mathrm{H}$ NMR $(200 \mathrm{MHz}): \delta$ $2.04(1 \mathrm{H}, \mathrm{dd}, J 9.5$ and $0.5 \mathrm{~Hz}), 2.16(1 \mathrm{H}, \mathrm{d}, J 9.5 \mathrm{~Hz})$, $4.36(1 \mathrm{H}$, dd, $J 11.0$ and $0.5 \mathrm{~Hz}), 4.47(1 \mathrm{H}, \mathrm{d}, J$ $11.0 \mathrm{~Hz}), 6.85-6.93(2 \mathrm{H}, \mathrm{m}), 7.22-7.30(2 \mathrm{H}, \mathrm{m}) .{ }^{13} \mathrm{C}$ NMR $(50 \mathrm{MHz}): \delta 29.3\left(\mathrm{CBr}_{2}\right), 35.9\left(\mathrm{CH}_{2}\right), 40.6(\mathrm{CBr})$, $75.3\left(\mathrm{OCH}_{2}\right) .116 .4(2 \times \mathrm{CH}) .126 .7(\mathrm{C}) .129 .4(2 \times \mathrm{CH})$. $156.6(\mathrm{C})$.

The compound was also prepared using the Seyferth procedure $^{9}$ [ $5.3 \mathrm{~g}(84 \%)$ from $1 \mathrm{~d}(3.71 \mathrm{~g}, 15 \mathrm{mmol})$ and phenyl(tribromomethyl )mercury(II) (11.91 g, $22.5 \mathrm{mmol})$ in dry benzene $(50 \mathrm{ml})$ ].

1,1,2-Tribromo-2-(4-nitrophenoxy) methylcyclopropane (2e) was obtained from $1 \mathrm{e}$ and was isolated by flash chromatography [hexane-ethyl acetate $=9: 1$ ] and recrystallized from hexane-ethyl acetate $(9: 1)$ to give pale yellow crystals; m.p. $141-143^{\circ} \mathrm{C}$; yield: $2.2 \mathrm{~g}(10 \%)$. IR $\left(\mathrm{CHCl}_{3}\right)$ : 3070 (w), 3010 (br, w), 2915 (w), 1605 (m), 1590 (s), 1510 (s), 1490 (m), 1445 (w), 1415 (w), 1380 (w), 1340 (s), $1330(\mathrm{sh}) 1295(\mathrm{~m}), 1255(\mathrm{br}, \mathrm{s}), 1170(\mathrm{~m}), 1110(\mathrm{~m})$, $1035(\mathrm{~m}), 1005(\mathrm{~m}), 925(\mathrm{w}), 860(\mathrm{~m}), 840(\mathrm{w}) \mathrm{cm}^{-1} \cdot{ }^{1} \mathrm{H}$ NMR $(200 \mathrm{MHz}): \delta 2.08(1 \mathrm{H}$, dd, $J 9.7$ and $0.5 \mathrm{~Hz})$, $2.19(1 \mathrm{H}, \mathrm{d}, J 9.7 \mathrm{~Hz}), 4.49(1 \mathrm{H}, \mathrm{dd}, J 11.0$ and $0.5 \mathrm{~Hz})$, $4.62(1 \mathrm{H}, \mathrm{d}, J 11.0 \mathrm{~Hz}), 7.01-7.05(2 \mathrm{H}, \mathrm{m}), 8.21-8.25$ $(2 \mathrm{H}, \mathrm{m}) .{ }^{13} \mathrm{C}$ NMR $(50 \mathrm{MHz}): \delta 28.9\left(\mathrm{CBr}_{2}\right), 35.9$ $\left(\mathrm{CH}_{2}\right), 40.0(\mathrm{CBr}), 75.4\left(\mathrm{OCH}_{2}\right), 114.8(2 \times \mathrm{CH}), 125.8$ $(2 \times \mathrm{CH}), 142.1$ (C), 162.9 (C). MS [m/z (rel. int.)]: 433 $\left(18, M^{+}\right), 431\left(56, M^{+}\right), 429\left(57, M^{+}\right), 427\left(19, M^{+}\right)$, 350 (100), 292 (24), 291 (25), 245 (25), 243 (26), 212 (50), $189(6), 152(6), 139(11), 133(24), 131(26), 118$ (18), 107 (7), $76(9), 63$ (16), 51 (15).

The compound was also prepared using the Seyferth procedure $^{9}$ [5.0 g $(79 \%)$ from $1 \mathrm{~d}(3.87 \mathrm{~g}, 15 \mathrm{mmol})$ and phenyl(tribromomethyl)mercury(II) (11.91 g, $22.5 \mathrm{mmol})$ in dry benzene $(50 \mathrm{ml})]$.

Dibromocarbene addition 1f. When 1f was subjected to dibromocarbene under phase-transfer conditions according to the general procedure 2-bromo-3-[formyl(4methoxyphenyl)amino]propene was furnished. The crude product, a yellow oil, was essentially pure; yield:
$11.0 \mathrm{~g}(81 \%) . \mathrm{IR}\left(\mathrm{CHCl}_{3}\right): 3150(\mathrm{~m}), 3000(\mathrm{~m}), 2950(\mathrm{~m})$, $2930(\mathrm{~m}), 2830(\mathrm{~m}), 2245$ (s), 1785 (br, m), 1665 (br, s), $1580(\mathrm{~m}), 1505(\mathrm{~s}), 1460(\mathrm{~m}), 1440(\mathrm{~m}), 1355(\mathrm{~s}), 1245$ (s), $1215(\mathrm{~s}), 1180(\mathrm{~m}), 1170(\mathrm{sh}), 1045(\mathrm{~m}), 1035(\mathrm{~m})$, 1020 (sh), 980 (m), 965 (m), 910 (br, s), 835 (m), 645 (s) $\mathrm{cm}^{-1} .{ }^{1} \mathrm{H}$ NMR $(200 \mathrm{MHz}): \delta 3.82(3 \mathrm{H}, \mathrm{s}), 4.60$ $(2 \mathrm{H}, \quad \mathrm{t}, \quad J 1.1 \mathrm{~Hz}), \quad 5.54-5.56(1 \mathrm{H}, \mathrm{m}), \quad 5.70-5.73$ (1 H, m), 6.89-6.95 (2 H, m), 7.13-7.28 (2 H, m), 8.40 $(1 \mathrm{H}, \mathrm{s}) .{ }^{13} \mathrm{C}$ NMR $(50 \mathrm{MHz}): \delta 52.6\left(\mathrm{NCH}_{2}\right), 55.2$ $\left(\mathrm{OCH}_{2}\right), 114.6(2 \times \mathrm{CH}), 119.2\left(=\mathrm{CH}_{2}\right), 125.9(2 \times \mathrm{CH})$, $127.1(\mathrm{C}), 132.7(\mathrm{CBr}), 158.5(\mathrm{C}), 162.6(\mathrm{C}=\mathrm{O})$.

Ring opening of $\mathbf{2}$ under phase-transfer conditions; general procedure. To a mixture of a 1,1,2-tribromo-2-aryloxymethylcyclopropane ( $5 \mathrm{mmol}$ ), triethylbenzylammonium chloride (TEBA) $(0.2 \mathrm{~g})$, and ethanol $(0.92 \mathrm{~g}, 20 \mathrm{mmol})$ in dichloromethane $(15 \mathrm{ml})$, kept under nitrogen at room temperature, was added $50 \%$ aqueous sodium hydroxide $(3.20 \mathrm{~g}, 40 \mathrm{mmol})$. The resulting mixture was stirred at room temperature until all starting material had been consumed as shown by TLC. Water was added, the products were extracted with diethyl ether and worked up in the usual way. The products were isolated by flash chromatography. By employing this procedure the following products were obtained.

3,3-Diethoxy-4-phenoxy-1-butyne (3a) was obtained from 2a and was isolated as a yellow oil by flash chromatography [hexane-ethyl acetate $=95: 5]$; yield: $1.06 \mathrm{~g}$ (90\%). IR (film): $3300(\mathrm{~m}), 3075(\mathrm{~m}), 3040(\mathrm{~m}), 2980(\mathrm{~s})$, $2940(\mathrm{~m}), 2885(\mathrm{~m}), 2120(\mathrm{~m}), 1770(\mathrm{~m}), 1595(\mathrm{~s}), 1500$ (s), $1460(\mathrm{~m}), 1380(\mathrm{~m}), 1245(\mathrm{~s}), 1140(\mathrm{~s}), 1050$ (br, s), $960(\mathrm{~m}), 830(\mathrm{~m}), 760(\mathrm{~s}), 690(\mathrm{~s}) \mathrm{cm}^{-1} \cdot{ }^{1} \mathrm{H}$ NMR $(200 \mathrm{MHz}): \delta 1.20(6 \mathrm{H}, \mathrm{t}, J 7.1 \mathrm{~Hz}), 2.42(1 \mathrm{H}, \mathrm{s})$, 3.59-3.72 (4 H, m), 3.99 (2 H, s), 6.73-6.93 (3 H, m), 7.02-7.27 (2 H, m). ${ }^{13} \mathrm{C}$ NMR $(200 \mathrm{MHz}): \delta \quad 15.0$ $\left(2 \times \mathrm{CH}_{3}\right), \quad 58.9 \quad\left(2 \times \mathrm{OCH}_{2} \mathrm{CH}_{3}\right), \quad 70.8(\mathrm{HC}), \quad 73.9$ $\left(\mathrm{OCH}_{2}\right), 79.4(\mathrm{C}), 96.1(\overline{\mathrm{C}}), 115.0(2 \times \mathrm{CH}), 121.1(\mathrm{CH})$, $129.2(2 \times \mathrm{CH}), 158.5(\mathrm{C})$. In addition, $60 \mathrm{mg}(5 \%)$ of 1,1-diethoxy-4-phenoxy-2-butyne (4a) was isolated as a yellow oil. IR (film): 3065 (m), 3040 (m), 2985 (s), 2930 (m), $2885(\mathrm{~m}), 2225(\mathrm{w}), 1760(\mathrm{~m}), 1605$ (s), 1500 (s), $1220(\mathrm{~s}), 1145$ (s), $1050(\mathrm{~s}), 920(\mathrm{~m}), 760(\mathrm{~s}), 695(\mathrm{~s}) \mathrm{cm}^{-1}$. ${ }^{1} \mathrm{H}$ NMR $(200 \mathrm{MHz}): \delta 1.15(6 \mathrm{H}, \mathrm{t}, J 7.1 \mathrm{~Hz}), 3.35-3.64$ $(4 \mathrm{H}, \mathrm{m}), 4.67(2 \mathrm{H}, \mathrm{d}, J 1.4 \mathrm{~Hz}), 5.12(1 \mathrm{H}, \mathrm{t}, J 1.4 \mathrm{~Hz})$, 6.86-6.91 (3 H, m), 7.17-7.25 (2 H, m). ${ }^{13} \mathrm{C} \quad \mathrm{NMR}$ $(50 \mathrm{MHz}): \quad \delta \quad 14.9 \quad\left(2 \times \mathrm{CH}_{3}\right), \quad 55.2 \quad\left(\mathrm{OCH}_{2}\right), \quad 60.1$ $\left(2 \times \mathrm{OCH}_{2} \mathrm{CH}_{3}\right), 79.5(\mathrm{C}), 82.7(\mathrm{C}), 90.7(\mathrm{CH}), 114.6$ $(2 \times \mathrm{C} \overline{\mathrm{H}}), 121.0(\mathrm{CH}), 128.9(2 \times \mathrm{CH}), 157.4(\mathrm{C})$.

3,3-Diethoxy-4-(4-methylphenoxy)-1-butyne (3b) was obtained from $\mathbf{2 b}$ and was isolated as a yellow oil by flash chromatography [hexane-ethyl acetate $=95: 5$ ]; yield: $0.91 \mathrm{~g}(73 \%)$. IR (film): $3280(\mathrm{~m}), 2985(\mathrm{~m}), 2920$ $(\mathrm{m}), 2880(\mathrm{~m}), 2110(\mathrm{w}), 1620(\mathrm{~m}), 1515(\mathrm{~s}), 1260(\mathrm{~m})$, $1160(\mathrm{~m}), 1045(\mathrm{~m}), 965(\mathrm{~m}), 825(\mathrm{~m}) \mathrm{cm}^{-1} .{ }^{1} \mathrm{H}$ NMR (200 MHz): $\delta 1.19(6 \mathrm{H}, \mathrm{t}, J 7.1 \mathrm{~Hz}), 2.25(3 \mathrm{H}, \mathrm{s}), 2.43$ (1 H, s), 3.58-3.71 (4 H, m), $3.96(2 \mathrm{H}, \mathrm{s}), 6.73-6.77$ 
( $2 \mathrm{H}, \mathrm{m}), 6.94-6.98(2 \mathrm{H}, \mathrm{m}) .{ }^{13} \mathrm{C} \mathrm{NMR}(50 \mathrm{MHz}): \delta$ $15.0\left(2 \times \mathrm{CH}_{3}\right), 20.4\left(\mathrm{CH}_{3}\right), 58.5\left(2 \times \mathrm{OCH}_{2} \mathrm{CH}_{3}\right), 71.0$ $(\mathrm{H}-\mathrm{C}), 73.5\left(\mathrm{OCH}_{2}\right), 79.6(\mathrm{C}), 95.9(\mathrm{C}), 114.6(2 \times \mathrm{CH})$, $129.3(2 \times \mathrm{CH}), 129.4(\mathrm{C}), 156.5(\mathrm{C})$. In addition, $125 \mathrm{mg}$ $(10 \%)$ of 1,1-diethoxy-4-(4-methylphenoxy)-2-butyne (4b) was isolated as a yellow oil. IR (film): 3045 (m), 2990 (s), 2925 (s), 2250 (w), 1615 (m), 1580 (m), 1510 (s), 1230 (s), $925(\mathrm{~m}), 815(\mathrm{~s}), 715(\mathrm{~m}) \mathrm{cm}^{-1} .{ }^{1} \mathrm{H}$ NMR $(200 \mathrm{MHz}): \delta 1.16(6 \mathrm{H}, \mathrm{t}, J 7.1 \mathrm{~Hz}), 2.28(3 \mathrm{H}, \mathrm{s})$, $3.46-3.59$ (4 H, m), $4.63(2 \mathrm{H}, \mathrm{d}, J 1.4 \mathrm{~Hz}), 5.12(1 \mathrm{H}, \mathrm{t}$, $J 1.4 \mathrm{~Hz}), 6.74-6.78(2 \mathrm{H}, \mathrm{m}), 6.97-7.01(2 \mathrm{H}, \mathrm{m}) .{ }^{13} \mathrm{C}$ NMR $(50 \mathrm{MHz}): \delta 15.0\left(2 \times \mathrm{CH}_{3}\right), 20.4\left(\mathrm{CH}_{3}\right), 55.3$ $\left(\mathrm{OCH}_{2}\right), 60.1\left(2 \times \mathrm{OCH}_{2} \mathrm{CH}_{3}\right), 79.7(\mathrm{C}), 82.5(\mathrm{C}), 90.7$ $(\mathrm{CH}), 114.6(2 \times \mathrm{CH}), 129.4(2 \times \mathrm{CH}), 129.7(\mathrm{C}), 155.4$ (C).

3,3-Diethoxy-4-(4-methoxyphenoxy)-1-butyne (3c) was obtained from $\mathbf{2 c}$ and was isolated as a yellow oil by flash chromatography [hexane-ethyl acetate $=95: 5$ ]; yield: $1.05 \mathrm{~g}(79 \%)$. IR (film): $3280(\mathrm{~m}), 2970(\mathrm{~m}), 2940$ (m), $2900(\mathrm{~m}), 2260(\mathrm{w}), 1600(\mathrm{w}), 1515(\mathrm{~s}), 1240(\mathrm{~s})$, 1200 (sh), 1145 (s), 1060 (br, s), 970 (m), 825 (m) cm $\mathrm{cm}^{-1}$. ${ }^{1} \mathrm{H}$ NMR $(200 \mathrm{MHz}): \delta 1.19(6 \mathrm{H}, \mathrm{t}, J 7.1 \mathrm{~Hz}), 2.44$ (1 H, s), 3.54-3.78 (4 H, m), $3.71(3 \mathrm{H}, \mathrm{s}), 3.93(2 \mathrm{H}, \mathrm{s})$, 6.67-6.82 (4 H, m). ${ }^{13} \mathrm{C} \quad \mathrm{NMR} \quad(50 \mathrm{MHz}): \quad \delta \quad 15.1$ $\left(2 \times \mathrm{CH}_{3}\right), 54.9\left(\mathrm{OCH}_{3}\right), 58.4\left(2 \times \mathrm{OCH}_{2} \mathrm{CH}_{3}\right), 71.6$ $(\mathrm{CH}), 73.4\left(\mathrm{OCH}_{2}\right), 79.7(\mathrm{C}), 95.9(\mathrm{C}), 114.1(2 \times \mathrm{CH})$, $115.6(2 \times \mathrm{CH}), 152.7(\mathrm{C}), 153.9(\mathrm{C})$. In addition $130 \mathrm{mg}$ $(10 \%)$ of 1,1-diethoxy-4-(4-methoxyphenoxy)-2-butyne (4c) was isolated as a yellow oil. IR (film): 3045 (w), 2970 (s), 2940 (m), 2890 (m), 1600 (w), 1500 (s), 1200 (br, s), 1160 (s), 1050 (br, s), 830 (s) cm $\mathrm{cm}^{-1} .{ }^{1} \mathrm{H}$ NMR (200 MHz): $\delta 1.16(6 \mathrm{H}, \mathrm{t}, J 7.1 \mathrm{~Hz}), 3.35-3.67(4 \mathrm{H}, \mathrm{m})$, $3.71(3 \mathrm{H}, \mathrm{s}), 4.61(2 \mathrm{H}, \mathrm{d}, J 1.4 \mathrm{~Hz}), 5.12(1 \mathrm{H}, \mathrm{t}, J$ $1.4 \mathrm{~Hz}), 6.68-6.84(4 \mathrm{H}, \mathrm{m}) .{ }^{13} \mathrm{C} \mathrm{NMR}(50 \mathrm{MHz}): \delta$ $14.9\left(2 \times \mathrm{CH}_{3}\right), \quad 54.9\left(\mathrm{OCH}_{3}\right), \quad 56.0\left(\mathrm{OCH}_{2}\right), 60.1$ $\left(2 \times \mathrm{OCH}_{2} \mathrm{CH}_{3}\right), 79.9(\mathrm{C}), 82.6(\mathrm{C}), 90.8(\mathrm{CH}), 114.2$ $(2 \times \mathrm{CH}), 115.8(2 \times \mathrm{CH}), 151.6(\mathrm{C}), 154.2(\mathrm{C})$.

3,3-Diethoxy-4-(4-chlorophenoxy)-I-butyne (3d) was obtained from $2 \mathbf{d}$ and was isolated by flash chromatography [hexane-ethyl acetate $=95: 5]$; yield: $1.08 \mathrm{~g}$ (80\%). IR (film): $3300(\mathrm{~m}), 2985$ (s), 2930 (m), $2900(\mathrm{~m})$, $2120(\mathrm{~m}), 1760(\mathrm{~m}), 1595(\mathrm{~m}), 1495(\mathrm{~s}), 1395(\mathrm{~m}), 1280$ (s), 1245 (s), 1150 (s), 1070 (s), 1060 (sh), 960 (m), 815 (s), $670(\mathrm{~m}) \mathrm{cm}^{-1} .{ }^{1} \mathrm{H}$ NMR $(200 \mathrm{MHz}): \delta 1.19(6 \mathrm{H}, \mathrm{t}$, $J 7.0 \mathrm{~Hz}), 2.48(1 \mathrm{H}, \mathrm{s}), 3.54-3.75(4 \mathrm{H}, \mathrm{m}), 3.97(2 \mathrm{H}$, s), 6.79-6.87 $(2 \mathrm{H}, \mathrm{m}), 7.13-7.28(2 \mathrm{H}, \mathrm{m}) .{ }^{13} \mathrm{C} \mathrm{NMR}$ $(50 \mathrm{MHz}): \delta 15.1\left(2 \times \mathrm{CH}_{3}\right), 58.7\left(2 \times \mathrm{OCH}_{2} \mathrm{CH}_{3}\right), 71,4$ $(\mathrm{H}-\mathrm{C}), 73.9\left(\mathrm{OCH}_{2}\right), 79.3(\mathrm{C}), 95.8(\mathrm{C}), \overline{1} 16.0(2 \times \mathrm{CH})$, $125.9(\mathrm{C}), 129.1(2 \times \mathrm{CH}), 157.0(\mathrm{C})$. In addition $27 \mathrm{mg}$ (2\%) of 1,1-diethoxy-4-(4-chlorophenoxy)-2-butyne (4d) was isolated as a yellow oil. IR (film): $3070(\mathrm{w}), 2980$ (s), $2930(\mathrm{~m}), 2880(\mathrm{~m}), 2010(\mathrm{w}), 1870(\mathrm{w}), 1600(\mathrm{~m})$, $1500(\mathrm{~s}), 1450(\mathrm{~m}), 1350(\mathrm{~m}), 1220(\mathrm{~s}), 1100$ (br, s), 910 (m), $820(\mathrm{~s}), 795(\mathrm{~m}), 760(\mathrm{~m}), 620(\mathrm{~s}) \mathrm{cm}^{-1} .{ }^{1} \mathrm{H}$ NMR (200 MHz): $\delta 1.16(6 \mathrm{H}, \mathrm{t}, J 7.1 \mathrm{~Hz}), 3.38-3.67(4 \mathrm{H}, \mathrm{m})$, $4.67(2 \mathrm{H}, \mathrm{d}, J 1.4 \mathrm{~Hz}), 5.12(1 \mathrm{H}, \mathrm{t}, J 1.4 \mathrm{~Hz}), 6.81-6.89$
(2 H, m), 7.16-7.24 (2 H, m). ${ }^{13} \mathrm{C} \mathrm{NMR} \mathrm{(50} \mathrm{MHz):} \delta$ $14.9\left(2 \times \mathrm{CH}_{3}\right), 55.5\left(\mathrm{OCH}_{2}\right), 60.2\left(2 \times \mathrm{OCH}_{2} \mathrm{CH}_{3}\right), 78.8$ (C), $83.3(\mathrm{C}), 90.6(\mathrm{CH}), 116.0(2 \times \overline{\mathrm{CH}}), 126.3(\mathrm{C})$, $129.0(2 \times \mathrm{CH}), 155.9(\mathrm{C})$.

Reaction of 2c with dry ethanol-DBU. The reaction was performed with $0.83 \mathrm{~g}(2.0 \mathrm{mmol})$ of $2 \mathrm{c}$ as described in the literature. ${ }^{2}$ After work-up the IR and ${ }^{1} \mathrm{H}$ NMR spectra of the crude product mixture were recorded. The following peaks due to $3 \mathrm{c}$ were observed: IR (film): 3282 , $2258 \mathrm{~cm}^{-1}$. ${ }^{1} \mathrm{H}$ NMR (200 MHz): $\delta 2.45$ (s).

Reaction of $2 \mathbf{c}$ with isopropyl alcohol under phase-transfer conditions. The reaction was performed with $0.83 \mathrm{~g}$ $(2.0 \mathrm{mmol})$ of $\mathbf{2 c}$ as described in the literature. ${ }^{2}$ Work-up in the usual way and evaporation of the volatile components gave $0.44 \mathrm{~g}(75 \%)$ of a $19: 1$ mixture of 1,1-diisopropoxy-4-(4-methoxyphenoxy)-2-butyne (6) and 3,3-diisopropoxy-4-(4-methoxyphenoxy)-1-butyne (7), which were not separated, but characterised as a mixture by the following spectroscopic data. IR (film): 3290 (m), 2990 (s), 2940 (s), 2840 (s), 2260 (w), 2070 (w), 1595 (m), $1505(\mathrm{~s}), 1465(\mathrm{~s}), 1385$ (s), 1350-1310 (s), 1050-1025 (s), 920 (s), 835 (s), 745 (s) $\mathrm{cm}^{-1} .{ }^{1} \mathrm{H}$ NMR (200 MHz): $\delta 1.18(12 \mathrm{H}, \mathrm{d}, J 6.2 \mathrm{~Hz}), 2.49(0.05 \mathrm{H}, \mathrm{s}), 3.82(3 \mathrm{H}$, s), $4.04(2 \mathrm{H}$, sept, $J 6.2 \mathrm{~Hz}), 4.57(0.10 \mathrm{H}, \mathrm{d}, J 1.4 \mathrm{~Hz})$, $4.67(1.90 \mathrm{H}$, br s), $5.35(0.95 \mathrm{H}, \mathrm{t}, J 1.4 \mathrm{~Hz}), 6.65-6.90$ $(4 \mathrm{H}, \mathrm{m}) \cdot{ }^{13} \mathrm{C}$ NMR $(50 \mathrm{MHz}): \delta 22.3\left(2 \times \mathrm{CH}_{3}\right), 22.6$ $\left(2 \times \mathrm{CH}_{3}\right), 55.4\left(\mathrm{OCH}_{3}\right), 56.4\left(\mathrm{OCH}_{2}\right.$, minor $), 56.6$ $\left(\mathrm{OCH}_{2}\right.$, major), $67.9(2 \times \mathrm{CH}), 83.4(\mathrm{C}), 88.6(\mathrm{C}), 114.8$ $(2 \times \mathrm{CH}), 115.9(2 \times \mathrm{CH}), 151.6(\mathrm{C}), 154.3(\mathrm{C})$.

Preparation of cis-1,1,2-trichloro-2-diethoxymethyl-3methylcyclopropane (8). To a vigorously stirred mixture of $(E)$ - and $(Z)$-1-ethoxy-1-propene $(43.0 \mathrm{~g}, 0.5 \mathrm{~mol})$, chloroform (with $1 \%$ ethanol) $(238.8 \mathrm{~g}, 4.0 \mathrm{~mol})$, and triethylbenzylammonium chloride (TEBA, ca. $0.5 \mathrm{~g}$ ) in dichloromethane (ca. $50 \mathrm{ml}$ ) was added dropwise a $50 \%$ aqueous solution of $\mathrm{NaOH}(120.0 \mathrm{~g}, 1.5 \mathrm{~mol})$. The resulting mixture was stirred at $0^{\circ} \mathrm{C}$ overnight and worked up in the usual way. The product, a cis/trans mixture of 1,1-dichloro-2-ethoxy-3-methylcyclopropane, was isolated by distillation, b.p. $48-50^{\circ} \mathrm{C} / 18 \mathrm{mmHg}$ (lit. ${ }^{22}$ b.p. $44-46{ }^{\circ} \mathrm{C} / 12 \mathrm{mmHg}$ ). The yield was $71.5 \mathrm{~g}$ (85\%).

Ring opening of the cis/trans mixture of 1,1-dichloro2-ethoxy-3-methylcyclopropane in a refluxing mixture of dry ethanol and dry pyridine according to Skattebøl ${ }^{22}$ gave $(Z)$-2-chloro-1,1-diethoxy-2-butene in $80 \%$ yield. The spectroscopic properties were in accordance with literature data. ${ }^{22}$

cis-1,1,2-Trichloro-2-diethoxymethyl-3-methylcyclopropane (8) was obtained by dropwise addition of a $50 \%$ aqueous solution of $\mathrm{NaOH}(64.0 \mathrm{~g}, 0.80 \mathrm{~mol})$ to a vigorously stirred mixture of (Z)-2-chloro-1,1-diethoxy-2butene $(17.9 \mathrm{~g}, 0.10 \mathrm{~mol})$, chloroform (with $1 \%$ ethanol) $(71.6 \mathrm{~g}, \quad 0.60 \mathrm{~mol})$, triethylbenzylammonium chloride (TEBA, ca. $1 \mathrm{~g}$ ) and dichloromethane $(60 \mathrm{ml})$. (An 
intense red colour appeared immediately the base was added and lasted during most of the addition.) The reaction mixture was stirred at $0^{\circ} \mathrm{C}$ overnight and worked up in the usual way. The product was purified by dryflash chromatography with hexane and isolated by distillation, b.p. $80-82^{\circ} \mathrm{C} / 1 \mathrm{mmHg}$. The yield was $15.4 \mathrm{~g}$ (59\%). IR: 2977 (s), 2931 (m), $2886(\mathrm{~m}), 1454(\mathrm{~m}), 1371$ (m), $1337(\mathrm{~m}), 1171$ (s), 1073 (s), $895(\mathrm{w}), 842(\mathrm{~m}) \mathrm{cm}^{-1}$. ${ }^{1} \mathrm{H}$ NMR (200 MHz): $\delta 1.21-1.41(9 \mathrm{H}, \mathrm{m}), 1.89(1 \mathrm{H}$, q, $J 6.5 \mathrm{~Hz}), 3.53-3.94(4 \mathrm{H}, \mathrm{m}), 4.52(1 \mathrm{H}, \mathrm{s}) .{ }^{13} \mathrm{C}$ NMR ( $50 \mathrm{MHz}): \delta 9.8\left(\mathrm{CH}_{3}\right), 15.0\left(\mathrm{CH}_{3}\right), 32.2\left(\mathrm{CH}_{3} \mathrm{CH}\right)$, $56.6(\mathrm{C}), 63.4\left(\mathrm{CH}_{2} \mathrm{O}\right), 63.7\left(\mathrm{CH}_{2} \mathrm{O}\right), 66.2(\mathrm{C}), 103.2$ $\left[\mathrm{CH}(\mathrm{OEt})_{2}\right]$.

Ring opening of $\mathbf{8}$ under phase-transfer conditions. The reaction was performed with $3.9 \mathrm{~g}(15 \mathrm{mmol})$ of $\mathbf{8}$ as described in the literature. ${ }^{1}$ (An intense red colour appeared immediately the base was added and lasted during most of the addition.) Product isolation by flash chromatography (hexane-EtOAc $=85: 15$ ) gave $3.0 \mathrm{~g}$ $(83 \%)$ of 4,4,5,5-tetraethoxy-2-pentyne (9). IR (film): 2970 (s), 2920 (s), 2870 (s), $2610(\mathrm{w}), 2230(\mathrm{~m}), 1725$ (m), $1695(\mathrm{~m}), 1575(\mathrm{~m}), 1470(\mathrm{~m}), 1430$ (s), 1375 (s), $1320(\mathrm{~s}), 1250(\mathrm{~s}), 1180-1060(\mathrm{~s}), 955(\mathrm{~m}), 915(\mathrm{~m}), 875$ (m), $800(\mathrm{~m}), 740(\mathrm{~m}), 660 \quad(\mathrm{~m}) \mathrm{cm}^{-1} .{ }^{1} \mathrm{H} \quad \mathrm{NMR}$ $(200 \mathrm{MHz}): \delta$ 1.13-1.28 (12 H, m), 1.87 (3 H, s), 3.62-3.89 (8 H, m), $4.35(1 \mathrm{H}, \mathrm{s}) .{ }^{13} \mathrm{C}$ NMR (50 MHz):

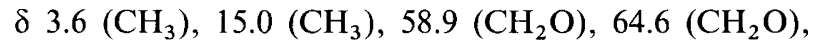
$73.7(\mathrm{C}), 83.1(\mathrm{C}), 98.5\left[\mathrm{C}(\mathrm{OEt})_{2}\right], 103.8\left[\mathrm{CH}(\mathrm{OEt})_{2}\right]$. MS: 199 (13), 171 (7), 141 (51), $125(9), 113$ (19), 103 (100), 97 (36), 85 (40), $75(66), 67$ (28), $53(6)$.

Acknowledgements. Financial support from Norsk Hydro and Research Council of Norway is most gratefully acknowledged. Thanks are also due to Professor Udo Brinker, University of Vienna, Austria and Professor Guy Bertrand, Laboratoire de Chimie de Coordination $\mathrm{du}$ CNRS, Toulouse, France for valuable discussions.

\section{References}

1. Sydnes, L. K. and Bakstad, E. Acta Chem. Scand. 50 (1996) 446.

2. Sydnes, L. K. and Bakstad, E. Acta Chem. Scand. 52 (1998) 1029.

3. Furniss, B. S., Hannaford, A. J., Smith, P. W. G. and Tatchell, A. R. Vogel's Textbook of Practical Organic Chemistry, 5th ed., Longman Scientific \& Technical, Harlow 1989 , p. 986.

4. Booth, S. E., Jenkins P. R. and Swain, C. J. J. Chem. Soc., Chem. Commun. (1991) 1248.

5. Parker, K. A. and Casteel, D. A. J. Org. Chem. 53 (1988) 2847.

6. Meyer, F. E., Parsons, P. J. and de Meijere, A. J. Org. Chem. 56 (1991) 6487.

7. Makosza, M. and Wawrzyniewicz, M. Tetrahedron Lett. (1969) 4659.

8. Makosza, M. and Fedorynski, M. Synth. Commun. 3 (1973) 305.

9. Seyferth, D., Burlitch, J. M., Minasz, R. J., Mui, J. Y.-P., Simmons, H. D. Jr., Treiber, A. J. H. and Dowd, S. R. J. Am. Chem. Soc. 87 (1965) 4259.

10. Seyferth, D. Acc. Chem. Res. 5 (1972) 65.

11. Müller, P. and Nguyen-Thi, H-C. Helv. Chim. Acta 67 (1984) 467.

12. Saunders, M. and Murray, R. W. Tetrahedron 6 (1959) 88.

13. Saunders, M. and Murray, R. W. Tetrahedron $11(1960) 1$.

14. Pierce, A. and Joullie, M. M. J. Org. Chem. 27 (1962) 2220.

15. Kocharian, A. K., Nilssen, A. V., Pettersen, A., Rømming, C. and Sydnes, L. K. Acta Chem. Scand. 42 (1988) 463 .

16. Sydnes, L. K., Pettersen, A., Drabløs, F. and Rømming, C. Acta Chem. Scand. 45 (1991) 902.

17. Nerdel, F., Buddrus, J., Windhoff, J., Brodowski, W., Klamann, D. and Ulm, K. Liebigs Ann. Chem. 710 (1967) 77.

18. Dehmlow, E. V. Chem. Ber. 101 (1968) 410.

19. Nazarov, S. I., Magerramov, M. N., Mamedov, S. M., Akhmedov, S. T. and Namazova, E. N. Zh. Org. Khim. 25 (1989) 1680.

20. Mori, M., Chiba, K., Okita, M., Kayo, I. and Ban, Y. Tetrahedron 41 (1985) 375.

21. Fröhlich, E. and Wedekind, E. Chem. Ber. 40 (1907) 1009.

22. Skattebøl, L. J. Org. Chem. 31 (1966) 1554.

Received November 2, 1998. 